\title{
ENFERMEDAD DE KIKUCHI EN JOVEN MASCULINO SIN ANTECEDENTES MÉDICOS. CAUSA INUSUAL DE FIEBRE DE ORIGEN DESCONOCIDO. PRESENTACIÓN DE CASO
}

\section{Kikuche disease in a Young male without previous medical history. Unusual cause of unknown fever. Case presentation}

\section{Elianet Castillo y Claudia Blanco}

Recibido: 26 de abril, $2020 \bullet$ Aprobado: 7 de agosto, 2020

Cómo citar: Castillo E, Blanco C. Enfermedad de Kikuchi en joven masculino sin antecedentes médicos. Causa inusual de fiebre de origen desconocido. Presentación de caso. cysa [Internet]. 15 de febrero de 2021 [citado 23 de febrero de 2021];5(1):117-21. Disponible en: https://revistas.intec.edu.do/index.php/cisa/article/view/2055

\section{Resumen}

Introducción: la enfermedad de Kikuchi, también llamada enfermedad Kikuchi-Fujimoto o linfadenitis necrotizante histiocítica es una condición benigna de etiología incierta que usualmente se presenta como fiebre de origen desconocido (FOD).

Objetivo: presentar un caso de un paciente joven que se ingresa en nuestro centro de salud con historia de fiebre de más de un mes, cefalea y adenopatías, que resultó ser la enfermedad de Kikuchi-Fujimoto.

Palabras clave: Kikuchi; Kikuchi-Fujimoto; linfadenitis necrotizante; fiebre de origen desconocido; República Dominicana.

\begin{abstract}
Introduction: Kikuchi's Disease, also called Kikuchi - Fujimoto Disease (KFD) or histiocytic necrotizing lymphadenitis is a benign condition with a vague etiology that usually presents itself as fever of unknown origin (UFO).

Objective: To present a case of a young patient that gets admitted to our healthcare center with one month history of fever, headache and adenopathy, that turned out to be Kikuchi-Fujimoto's Disease
\end{abstract}

Keywords: Kikuchi; Kikuchi-Fujimoto; necrotizing lymphadenitis; fever of unknown origin; Dominican Republic.

\footnotetext{
a Residente de $3^{\text {er }}$ año de Medicina Interna de Centro de Diagnóstico, Medicina Avanzada y Telemedicina (CEDIMAT), Santo Domingo, República Dominicana. ORCID: 0000-0002-6886-8311

Correo-e: elianetcastillo@cedimat.net
}

\footnotetext{
b Infectóloga-Internista, CEDIMAT, Santo Domingo, República Dominicana. ORCID: 0000-0002-6097-7388

Correo-e: claudiablanco@cedimat.net
} 


\section{Introducción}

La enfermedad de Kikuchi-Fujimoto es una condición benigna de causa desconocida caracterizada por fiebre y linfadenopatía, siendo una etiología frecuentemente pasada por alto en el estudio del paciente con fiebre de origen desconocido.

\section{Caso clínico}

En agosto del 2019, un masculino de 18 años de edad asiste a la emergencia con historia de 4 semanas de fiebre termometrada en $39^{\circ} \mathrm{C}$, varias a veces al día, refiere además cefalea leve y mareos.

Paciente sin antecedentes mórbidos, historia familiar no destacable, es estudiante que reside en zona urbana, antecedentes tóxicos, viajes, contacto con animales, personas enfermas, picaduras por artrópodos negados. Refiere historia de sexo casual con protección un mes previo al inicio de los síntomas y en la revisión por sistemas pérdida de peso de aproximadamente 15 libras desde el inicio de la fiebre.

En la evaluación clínica inicial, encontramos paciente alerta, febril, mucosa oral seca y presencia de masa en región lateral derecha del cuello, de bordes regulares, suave, dolorosa, de tamaño aproximado de 6 cm cuyo tiempo de evolución es de tres semanas. Signos vitales normales, no soplos, visceromegalias, eritemas o estigmas de sangrado, no datos de irritación meníngea; se decide su admisión al centro.

En las analíticas iniciales, el hemograma presentó solo anemia microcítica hipocrómica leve, electrolitos, función hepática y renal dentro de los valores normales, procalcitonina negativa, examen de orina no patológico, radiografía de tórax simple sin hallazgos, gota gruesa e IGRA negativos y serología para Leptospira, Hepatitis A, Hepatitis B, Hepatitis C, HTLV 1, HIV, Toxoplasmosis, Epstein-Barr y Citomegalovirus, resultando solo la IgG de Citomegalovirus positiva. Se toman muestras adicionales para extendido de sangre periférica que reporta solo la presencia de eliptocitos y ovalocitos, así como hemocultivos que se reportan sin crecimiento de patógenos.

Se evidencian en los laboratorios datos de respuesta inflamatoria sistémica presentando elevados la Ferritina $610 \mathrm{ng} / \mathrm{ml}$, Eritrosedimentación $27 \mathrm{~mm} / \mathrm{hr}$, proteína $\mathrm{C}$ reactiva $4.5 \mathrm{mg} / \mathrm{L}$ y LDH $870 \mathrm{U} / \mathrm{L}$.

Se realiza ecocardiograma que se reporta sin evidencia de vegetaciones y tomografía de cráneo, cuello, tórax, abdomen y pelvis contrastada que reportan como resultado a destacar la presencia de múltiples adenopatías en todos los espacios del cuello, en el nivel II B con centro necrótico evidente y engrosamiento inespecífico del timo sugestivo de hiperplasia: figura 1.
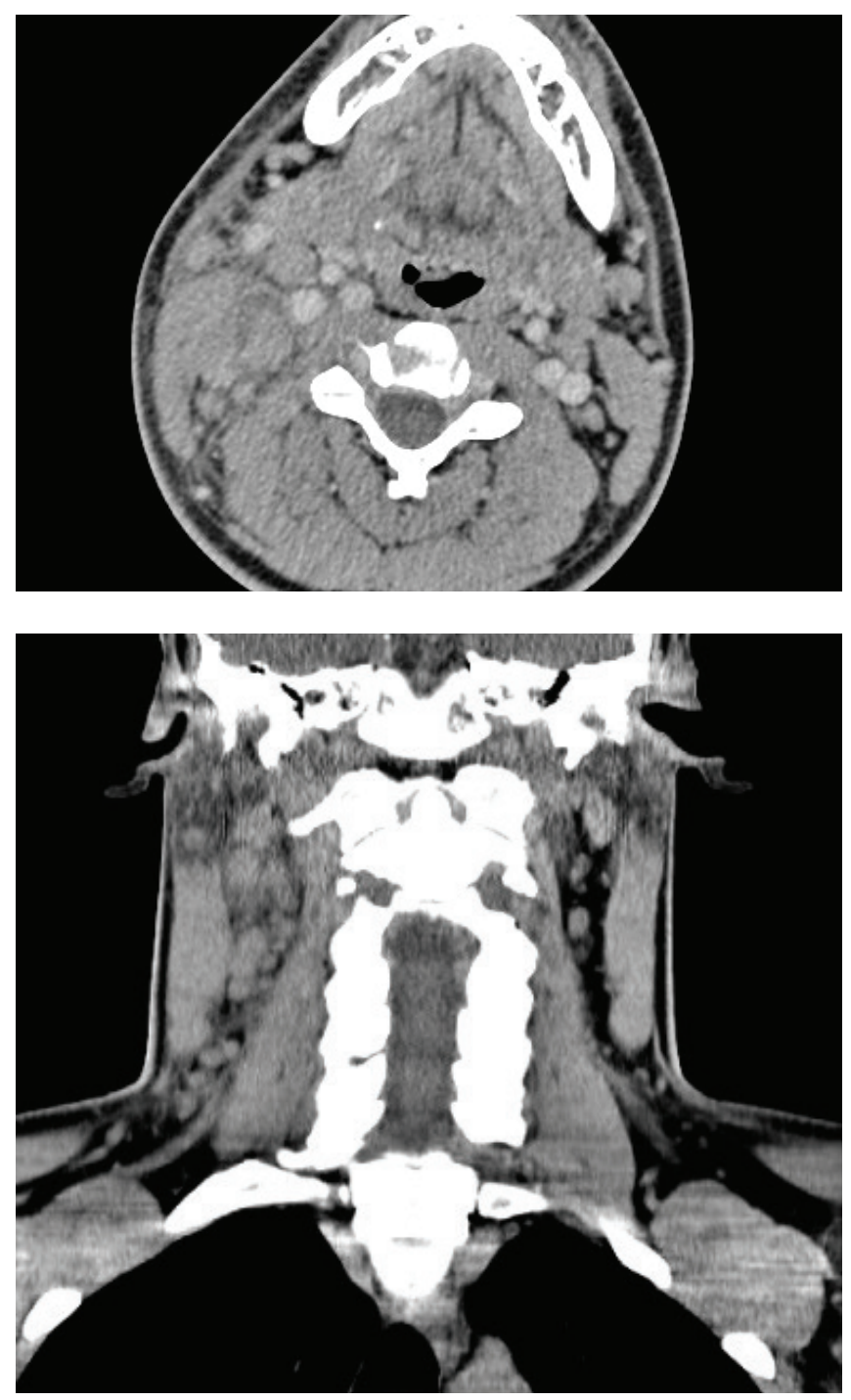

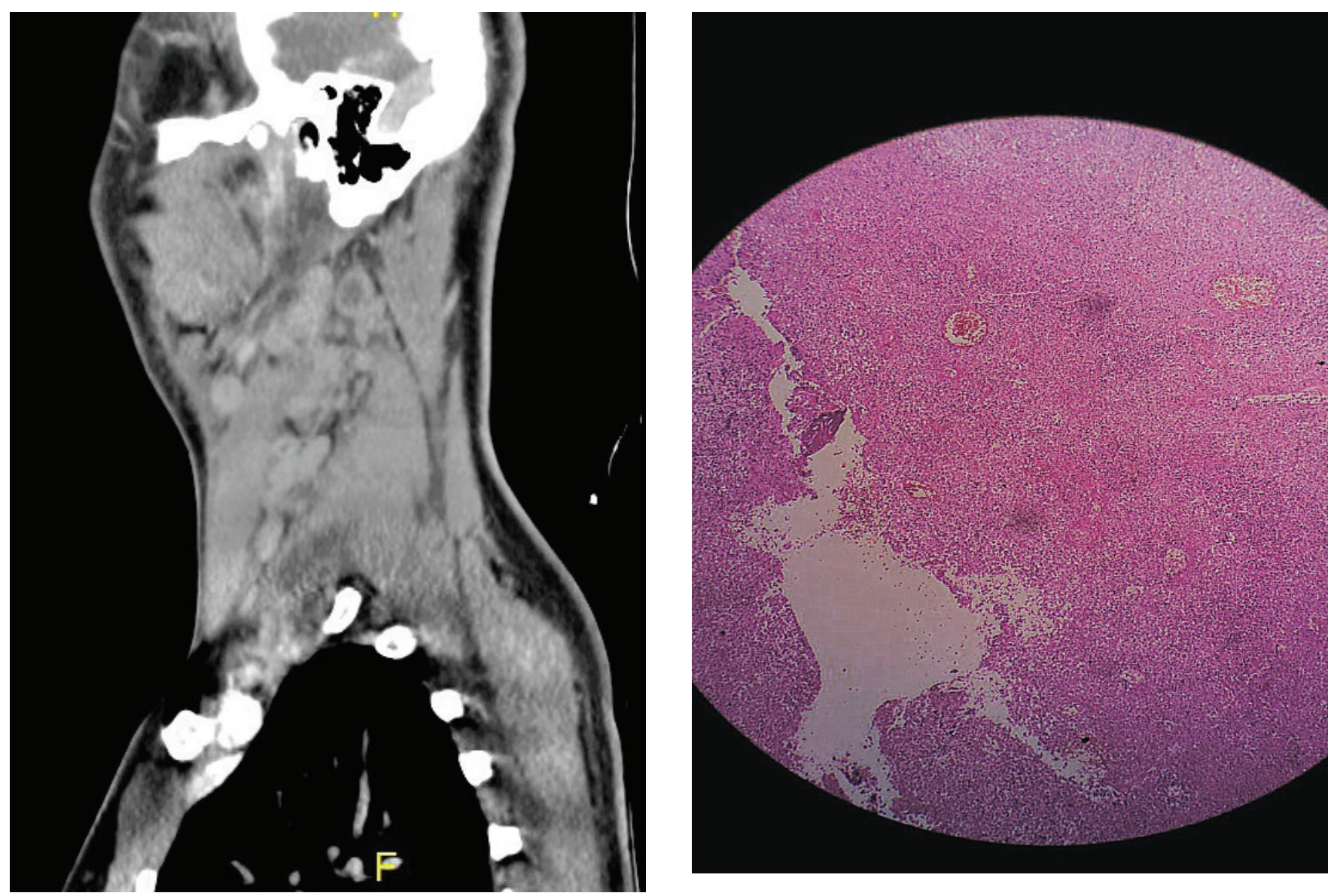

Figura 1. Cortes tomográficos axial, coronal y sagital mostrando múltiples adenopatías, algunas con centro necrótico.

Fuente: imágenes tomadas del Sistema de almacenamiento y distribución de imagen, (PACS, por sus siglas en inglés) del centro.

El paciente durante su hospitalización continuó presentando fiebre diariamente y no evidenció síntomas o signos nuevos que pudiesen orientar hacia una etiología específica, por lo que se decide tomar muestra excisional de ganglio linfático, más abordable para cultivo y patología; el cultivo para micobacterias se reporta sin crecimiento luego de 8 semanas y biopsia del tejido reporta amplias zonas de necrosis, histiocitos sin presencia de reacción granulomatosa e inmunoblastos atípicos consistentes con una linfadenitis necrotizante histiocítica con atipia de ganglios linfáticos cervicales acorde con la Enfermedad de Kikuchi-Fujimoto: figura 2.

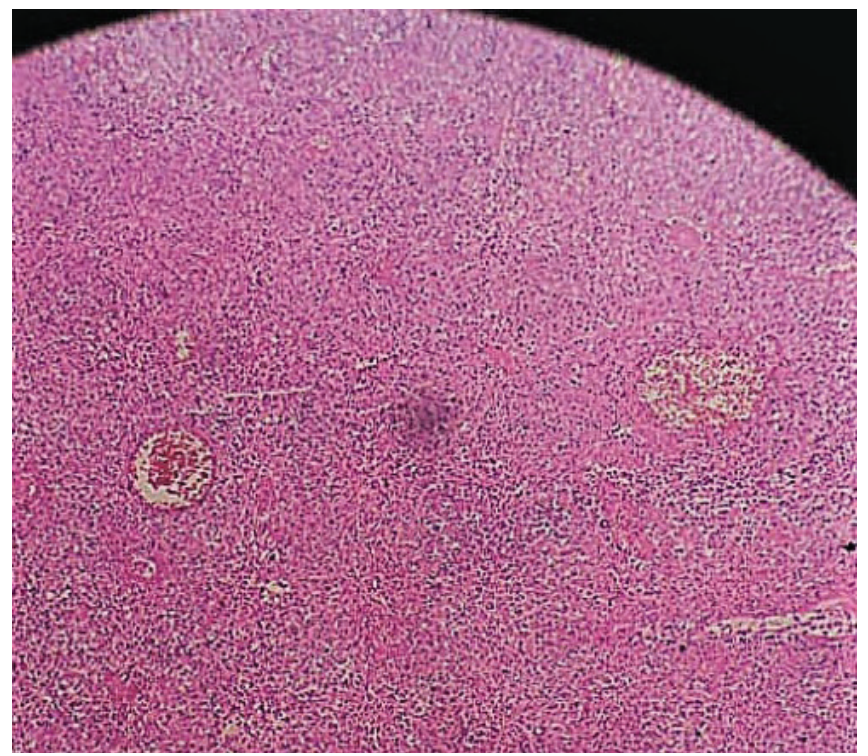

Figura 2. Patología del ganglio linfático mostrando amplias zonas de necrosis, histiocitos sin presencia de reacción granulomatosa.

Fuente: imágenes tomadas del Laboratorio de Patología del centro. 
El paciente fue egresado presentando mejoría de la fiebre y regresión de las adenopatías sin tratamiento específico a las pocas semanas.

\section{Discusión}

La enfermedad de Kikuchi-Fujimoto fue reportada por primera vez en Japón en $1972{ }^{1}$

Es una condición más frecuentemente encontrada en la población japonesa o del Asia del este. Extrapolando una serie de casos, la proporción hombre-mujer es prácticamente igual con una leve predominancia femenina. La mayoría de los pacientes tienen menos de 30 años de edad con una media de 25.5 a 30 años. $^{2}$

Infección por Yersinia Enterolítica, Brucelosis, Bartonella henselae, Entamoeba histolytica, Mycobacterium szulgai, Toxoplasma gondii, virus del Epstein-Barr, virus del herpes, citomegalovirus, parvovirus, parainfluenza, rubeola, hepatitis B, HTLV 1, HIV y dengue han sido todos implicados en la etiología de KFD. Ninguno ha sido encontrado consistentemente asociado a esta condición. ${ }^{3-4}$

Los síntomas y signos más comunes son linfadenopatía, fiebre, rash, artritis, fatiga y hepatoesplenomegalia. ${ }^{5} \mathrm{La}$ fiebre es prolongada variando entre 1 a 7 semanas, con temperaturas entre 38.6 a 40.5 grados Celsius. ${ }^{6} \mathrm{El}$ involucramiento de los ganglios linfáticos usualmente es cervical y localizado. ${ }^{7-8}$. Otros síntomas menos comunes son dolor de cabeza, náuseas, vómitos, malestar, pérdida de peso, artralgias, mialgias, sudoración nocturna, dolor torácico/ abdominal y síntomas asociados con enfermedad autoinmune. ${ }^{9}$

Actualmente, el único método fidedigno es la examinación histológica de una biopsia excisional de un ganglio linfático. Las pruebas de sangre clásicamente solo muestran una leve neutropenia con leve elevación de la proteína $\mathrm{C}$ reactiva $(\mathrm{PCR})$ y la Eritrosedimentación (ESR). ${ }^{8}$
En un estudio ${ }^{10}$, se encontraron las siguientes apariencias típicas en tomografía:

- Linfadenopatía homogénea múltiple involucrando los niveles II al V.

- $94 \%$ eran pequeñas, menores de $2.5 \mathrm{~cm}$, esto permitía la diferenciación del linfoma, que típicamente produce pocas adenopatías, pero más grandes.

- Infiltración perinodal y necrosis es comúnmente encontrada.

Los hallazgos histológicos son predominancia leve de los linfocitos CD8, infiltración linfohistiocítica y cariorrexis no neutrofilica. ${ }^{8}$ También se ha encontrado la presencia de áreas de necrosis sin infiltrado neutrófico asociado con una predominancia de histiocitos, siendo estos el tipo de célula mayor. ${ }^{10}$

Es una condición autolimitada, que raramente requiere tratamiento específico en la mayoría de los casos. En pacientes con síntomas neurológicos o en aquellos casos en donde KFD se encuentra asociado a otra condición médica, inmunosupresión con esteroides aparenta mejorar rápidamente la condición del paciente. ${ }^{8}$ Se ha estimado una tasa de recurrencia de $3 \%-4.5 \%$ y la mortalidad es extremadamente rara. ${ }^{11}$ Los pacientes con KFD deberían ser seguidos regularmente por años, para detectar la posible evolución a Lupus Eritematoso Sistémico. ${ }^{12}$

Es una condición singular de la cual todavía no se tiene la etiología, diagnóstico y tratamiento totalmente dilucidado por lo que un alto índice de sospecha es necesario para el diagnóstico e individualizar el plan de tratamiento de acuerdo a las características del paciente es la norma. 


\section{Bibliografía}

1. Dalton J, Shaw R, Democratis J. Kikuchi-Fujimoto disease. The Lancet. 2014Mar22;383 (9922):1098.

2. Lin H. Kikuchi's disease: a review and analysis of 61 cases. Otolaryngology - Head and Neck Surgery. 2003;128(5):650-3.

1. Hollingsworth $\mathrm{H}$. An investigation of the viral pathogenesis of Kikuchi-Fujimoto disease: lack of evidence for Epstein-Barr virus or human herpesvirus type 6 as the causative agents. Archives of Pathology and Laboratory Medicine. 1994;118(2):134-40.

2. Huh J, Chi HS, Kim SS, Gong G. A study of the viral etiology of histiocytic necrotizing lymphadenitis (Kikuchi-Fujimoto disease). Journal of Korean Medical Science. 1998;13(1):27.

3. Kucukardali, Y, Emrullah S, Erdogan K, Oral O, Sukru Y, y Mustafa K. «Kikuchi-Fujimoto Disease: Analysis of 244 Cases». Clinical Rheumatology. 2017;26(1):50-4.

4. Lee BC, Patel R. Kikuchi-Fujimoto disease: a 15-year analysis at a children's hospital in the UnitedStates. Clinical Pediatrics. 2013;52(1):92-5
5. Kuo T-T. A Clinicopathologic Study of 79 Cases with an Analysis of Histologic Subtypes, Immunohistology, and DNA Ploidy. The American Journal of Surgical Pathology. 1995;19(7): 798-809.

6. Dorfman RF, Berry GJ. Kikuchi's histiocytic necrotizing lymphadenitis: an analysis of 108 cases with emphasis on differential diagnosis. Seminars in Diagnostic Pathology. 1988 Nov;5(4):329-45

7. Poulouse V, Chiam P, Poh W. Kikuchi's disease: a Singapore case series. Singapore Medical Journal 2005;46:229-32.

8. Veer V, Lim A, Issing W. «Kikuchi-Fujimoto Disease: A Case Report and Literature Review». Case Reports in Otolaryngology. 2012;(2012):1-5.

9. Jayanti K, Shalini S, Vijay K, Sanjay K, Pratap S. "Kikuchi-Fujimoto Disease - A Clinical Enigma: Rare Two Case Reports». Journal of Advanced Research in Medicine. 2020;6(3):12-5.

10. Sopeña B, Rivera A, Vázquez-Triñanes C et al. Autoimmune manifestations of Kikuchi disease. Seminars in Arthritis and Rheumatism. 2012;41:900-6. 